\title{
The PrEPARE Pretoria Project: protocol for a cluster-randomized factorial-design trial to prevent HIV with PrEP among adolescent girls and young women in Tshwane, South Africa
}

Wendee M. Wechsberg ${ }^{1,2,3,4^{*}}$ (D), Felicia A. Browne ${ }^{1,5}$, Jacqueline Ndirangu ${ }^{1}$, Courtney Peasant Bonner ${ }^{1}$, Alexandra M. Minnis ${ }^{6,7}$, Laura Nyblade ${ }^{8}$, Ilene S. Speizer ${ }^{2}$, Brittni N. Howard ${ }^{1}$, Bronwyn Myers ${ }^{9}$ and Khatija Ahmed ${ }^{10}$

\begin{abstract}
Background: Despite increased prevention efforts, HIV remains the leading cause of death among adolescent girls and young women in South Africa. Although research indicates important determinants of HIV acquisition at the individual and interpersonal levels, structural-level stigma and discrimination continue to be critical barriers to reaching and retaining this key population for HIV prevention and sexual and reproductive health services. Innovative and multilevel interventions are needed that can address the intersectional structural and gender issues that young women face, including stigma, alcohol and drug use, gender-based violence, and other risk factors when seeking health services. Oral pre-exposure prophylaxis (PrEP) taken daily has been found to be an effective biomedical HIV prevention tool. Testing a comprehensive gender-focused biobehavioral HIV prevention intervention that is inclusive of social ecological determinants, such as stigma and discrimination reduction in clinics, is critical for reducing HIV among adolescent girls and young women.

(Continued on next page)
\end{abstract}

\footnotetext{
* Correspondence: wmw@rti.org

'Substance Use, Gender, and Applied Research Program, RTI International, 3040 E. Cornwallis Road, Research Triangle Park, NC 27709-2194, USA

${ }^{2}$ Maternal and Child Health, University of North Carolina Gillings School of Global Public Health, 135 Dauer Drive, Chapel Hill, NC 27599, USA

Full list of author information is available at the end of the article
}

(c) The Author(s). 2020 Open Access This article is licensed under a Creative Commons Attribution 4.0 International License, which permits use, sharing, adaptation, distribution and reproduction in any medium or format, as long as you give appropriate credit to the original author(s) and the source, provide a link to the Creative Commons licence, and indicate if changes were made. The images or other third party material in this article are included in the article's Creative Commons licence, unless indicated otherwise in a credit line to the material. If material is not included in the article's Creative Commons licence and your intended use is not permitted by statutory regulation or exceeds the permitted use, you will need to obtain permission directly from the copyright holder. To view a copy of this licence, visit http://creativecommons.org/licenses/by/4.0/ The Creative Commons Public Domain Dedication waiver (http://creativecommons.org/publicdomain/zero/1.0/) applies to the data made available in this article, unless otherwise stated in a credit line to the data. 
(Continued from previous page)

Methods: This project involves both a Community Collaborative Board and a Youth Advisory Board in helping to adapt the Young Women's Health CoOp intervention and the Health Policy Project (HPP) Stigma and Discrimination (S\&D) reduction training curriculum to the setting and population. This study uses a two-by-two factorial design with stratified randomization of 12 clinics, each with distinct catchment areas. The Young Women's Health CoOp addresses substance use, sexual risk, violence prevention and sexual negotiation, condom demonstration, and problem solving with the following additions: knowledge of PrEP, the importance of PrEP adherence, and sexual and reproductive health. Adolescent girls and young women will be assessed with behavioral and biological measures at baseline, 3-, 6- and 9-month follow-up. The S\&D reduction training is provided for all staff in the clinics randomized to this condition. Clinic staff will be surveyed at baseline, 4- and 8-month follow-up. We will recruit 900 AGYW from communities in the 12 clinic catchment areas.

Discussion: The study findings, if efficacious across the outcomes, will be incorporated into the gender-focused HIV prevention intervention toolkit and disseminated to inform multilevel prevention approaches.

Trial registration: ClinicalTrials.gov. Identifier: NCT04048551 (Recruiting). Registered: August 7, 2019 (Retrospectively registered).

Keywords: HIV, Sexual and reproductive health (SRH), Stigma, Health Policy Project Stigma and Discriminationreduction training (HPP S\&D), Clinic staff, Young Women's Health CoOp (YWHC), Alcohol and drug use, Genderbased violence, Adaptation

\section{Background}

Despite important progress globally to reduce HIV incidence, adolescent girls and young women (AGYW) continue to be disproportionately at risk of HIV acquisition [1]. In South Africa, new HIV infections are concentrated among AGYW between 15 to 24-this group accounts for over $25 \%$ of new in-country infections, three times the rate of young men in this age group [2]. Additionally, estimates suggest that in some areas of South Africa, AGYW have an HIV prevalence of $4 \%$, which increases to $24 \%$ for young women aged 20 to 24 [2]. This highlights the high incidence of HIV in late adolescence and early adulthood and the critical need for prevention in this age group.

Research indicates important determinants of HIV acquisition at the individual and interpersonal levels. However, structural-level stigma and discrimination (S\&D) continues to be a critical barrier to reaching and retaining this key population for HIV prevention and sexual and reproductive health (SRH) services [3-6]. AGYW who engage in high-risk sex are aware of their risk and desire healthcare services, but service access is often impeded by poor treatment by clinic staff $[6,7]$. In formative focus group discussions conducted in Tshwane for this study, AGYW expressed concerns about treatment by nurses when seeking SRH services and about confidentiality in clinics $[6,7]$. S\&D also prevents engagement and retention in HIV prevention services [8-12]. Anticipated and experienced HIV stigma extends to biomedical HIV prevention strategies, such as pre-exposure prophylaxis (PrEP), and is also a barrier to HIV prevention efforts [13-16]. Providing PrEP in clinics may not be effective if vulnerable AGYW are disinclined to visit clinics because of fear of $S \& D$ or patronizing treatment by clinic staff based on assumptions about their age and sexual activity. Consequently, S\&D training for clinic staff is essential to reduce barriers for AGYW to access services.

Intersectional stigma toward AGYW is the convergence of multiple stigmatized identities, such as age, health, gender, behavior, and socioeconomic status [17]. Because individuals' characteristics/identities are not in isolation, stigma needs to be addressed through multiple-level combination interventions [18]. Further, intersectional issues-including condomless sex and the lack of personal power or skills to negotiate safer sex, the need to conduct transactional sex, cross-generational sex, gender-based violence (GBV), substance use, and other risky behaviors-affect the likelihood that AGYW may acquire HIV during emerging adulthood [2, 19].

Globally, momentum is increasing to avert HIV infection among AGYW via the use of comprehensive strategies in the care continuum. The South African government acknowledges this need, as demonstrated by its involvement in the Determined, Resilient, Empowered, AIDS-free, Mentored and Safe (DREAMS) program, which aims to prevent HIV among vulnerable AGYW by empowering them through social asset building and by providing post-violence care, contraception through SRH services, and HIV prevention such as condoms and biomedical prevention such as PrEP [20].

\section{Oral PrEP: a female-controlled HIV prevention tool}

Oral PrEP, a combination of the antiretroviral (ARV) medications emtricitabine (FTC) and tenofovir disoproxil fumarate (TDF) taken daily, prevents HIV 
acquisition among individuals who are HIV-negative. It has been found to be effective among women in Africa $[21,22]$. It was approved for use by the U.S. Food and Drug Administration in 2012 [23] and the South African Government in 2015 [24].

Because of an inability to negotiate condom use or mutual monogamy [25-27], many AGYW are unable to protect themselves from HIV, even when they are particularly vulnerable to HIV transmission [2]. These AGYW may benefit from PrEP because it does not require partner involvement. Recent open-label demonstration projects that offered oral PrEP to young women [28], including some in South Africa, have demonstrated that PrEP is feasible among this age group [29]. However, placebo-controlled efficacy trials, such as the FemPrEP and VOICE trials found low PrEP adherence rates [30-32]. Although oral PrEP offers a promising HIV prevention strategy for AGYW, its effectiveness depends on high adherence [33]. To support PrEP uptake and adherence, an integrated HIV prevention program is needed that addresses multiple barriers and risk behaviors, with a focus on the individual, interpersonal, and structural levels, that may impede PrEP readiness, uptake, adherence, and engagement in safer sexual behaviors among AGYW who are HIV-negative [34].

\section{The adapted Young Women's Health CoOp}

The Women's Health CoOp (WHC), the original intervention, is an evidence-based woman-focused HIV prevention intervention grounded in empowerment and feminist theory to increase individual knowledge and skills to reduce substance use and increase personal power around HIV risk behaviors [35]. The WHC has been shown to be efficacious in several National Institutes of Health-funded studies in South Africa [36-39].

The Young Women's Health CoOp (YWHC) was originally adapted from a U.S. adolescent project and subsequently fielded in South Africa for a younger generation of women at risk for HIV $[4,19,40]$. The YWHC included the core elements of the most recent WHC addressing substance use, sexual risk, violence prevention and sexual negotiation, condom demonstration, and problem solving, including role-play practice. The next generation of the YWHC, which was adapted for the current study entitled, The PRevention, Empowering, and PRotEcting (PrEPARE) Project includes knowledge of PrEP, such as the importance of adherence and in-depth information on contraception and other SRH options. The added elements of PrEP and SRH material, along with assistance with accessing clinics for PrEP initiation and adherence and contraception, ensures that AGYW receive a more comprehensive HIV prevention program and SRH services.
Although the YWHC addresses the individual and interpersonal needs of AGYW who engage in risk behaviors, such as alcohol and drug use and condomless sex, it did not address the structural barriers, such as stigma and discrimination, that many AGYW face when accessing clinics for services. Consequently, the structural aim of this study addresses S\&D-reduction training in clinics.

\section{Addressing structural stigma and discrimination in clinics} The Health Policy Project (HPP) Health Facility HIVStigma and Discrimination reduction training curriculum addresses barriers that arise from S\&D. The HPP curriculum is based on a decade of implementation experience in Africa, South and South-East Asia, and the Caribbean [41]. It has been further adapted and implemented in a range of settings, most recently in Ghana [42], Tanzania [43, 44] and other regions of the world $[45,46]$. The curriculum provides participatory training modules that address three key actionable drivers of HIV stigma: lack of understanding of S\&D; fear of workplace HIV transmission; and clinic staff attitudes. For this study, the HPP curriculum was modified to address the lives and experiences of AGYW seeking SRH and PrEP services.

To decrease structural S\&D in clinics and increase AGYW's agency to protect themselves, a multilevel strategy is needed. The present study seeks to determine whether implementing a multilevel, woman-focused intervention for PrEP readiness, uptake, and adherence among vulnerable AGYW in Tshwane, South Africa, that includes S\&D reduction assessment and training in clinics, is a viable complement to South Africa's National Strategic plan for HIV, which includes a focus on AGYW [47]. It also seeks to address barriers to accessing SRH services among this vulnerable population by reducing health facility-wide $S \& D$.

\section{Methods/design \\ Aim and objectives}

This project comprises three specific aims. Aim 1, which was completed during the study's formative phase, involved identifying and randomizing eligible clusters (study clinics) and engaging community stakeholders through a Community Collaborative Board (CCB) and Youth Advisory Board (YAB) to assist in the adaptation of the YWHC and S\&D reduction training program to the South African geographic and cultural context. This study protocol is based on Aims 2 and 3, the experimental phase of the study. Aim 2 is to evaluate the impact of the HPP S\&D reduction training among clinic staff on the use of HIV and SRH services by AGYW, including PrEP, and staff attitudes and behaviors toward AGYW at 4- and 8-month follow-up. Aim 3 is to test the efficacy of a multilevel HIV prevention strategy that addresses 
structural (S\&D reduction), interpersonal (peer social support), and individual (personal agency, substance use, and GBV) factors on PrEP readiness, uptake, and adherence; HIV status; and other risk-taking behaviors at 3-, 6-, and 9-month follow-up among vulnerable AGYW. We plan to recruit 900 AGYW across 12 communities in clinic catchment areas for the experimental phase of study.

\section{Setting}

We conducted feasibility assessments of potential study sites in provincial and city clinics in Tshwane, South Africa; 15 clinics were selected as the final study clinics. Each assessment included engaging the clinic manager and requesting permission to conduct the assessment. The selected clinics had (1) community ward-based outreach teams (WBOTs), (2) comprehensive SRH services, and (3) designated youth-friendly nurses and/or services. Other criteria included (a) the jurisdiction of the cliniccity or provincial, as this determines the allocation of resources and infrastructure, (b) reported average number of AGYW visiting the clinic per month, and (c) clinic location (peri-urban or semi-rural community) within the
Tshwane district. As a result, the final sample consisted of 12 study clinics, 8 provincial clinics and 4 city clinics, with 3 additional clinics as backups if one or more of the selected clinics turns out not to be viable.

\section{Study design}

This cluster randomized trial utilizes a $2 \times 2$ factorial design [48] to assess the impact of S\&D reduction training and the YWHC intervention, resulting in four study conditions: (1) Clinic S\&D reduction training + PrEP/SRH + YWHC, (2) Clinic S\&D reduction training + PrEP/SRH only, (3) No Clinic S\&D reduction training + PrEP/SRH + YWHC, (4) No Clinic S\&D reduction training + PrEP/ SRH only (Fig. 1).

\section{Randomization}

Stratified randomization was conducted via SAS Software after clinic selection was finalized. The sample of 12 clinics was first grouped into three strata (peri-urban/ city, peri-urban/provincial, and semi-rural/provincial) based on geographic location type (peri-urban vs. semirural) and clinic administration type/jurisdiction (city vs. provincial), with four clinics in each stratum. Within

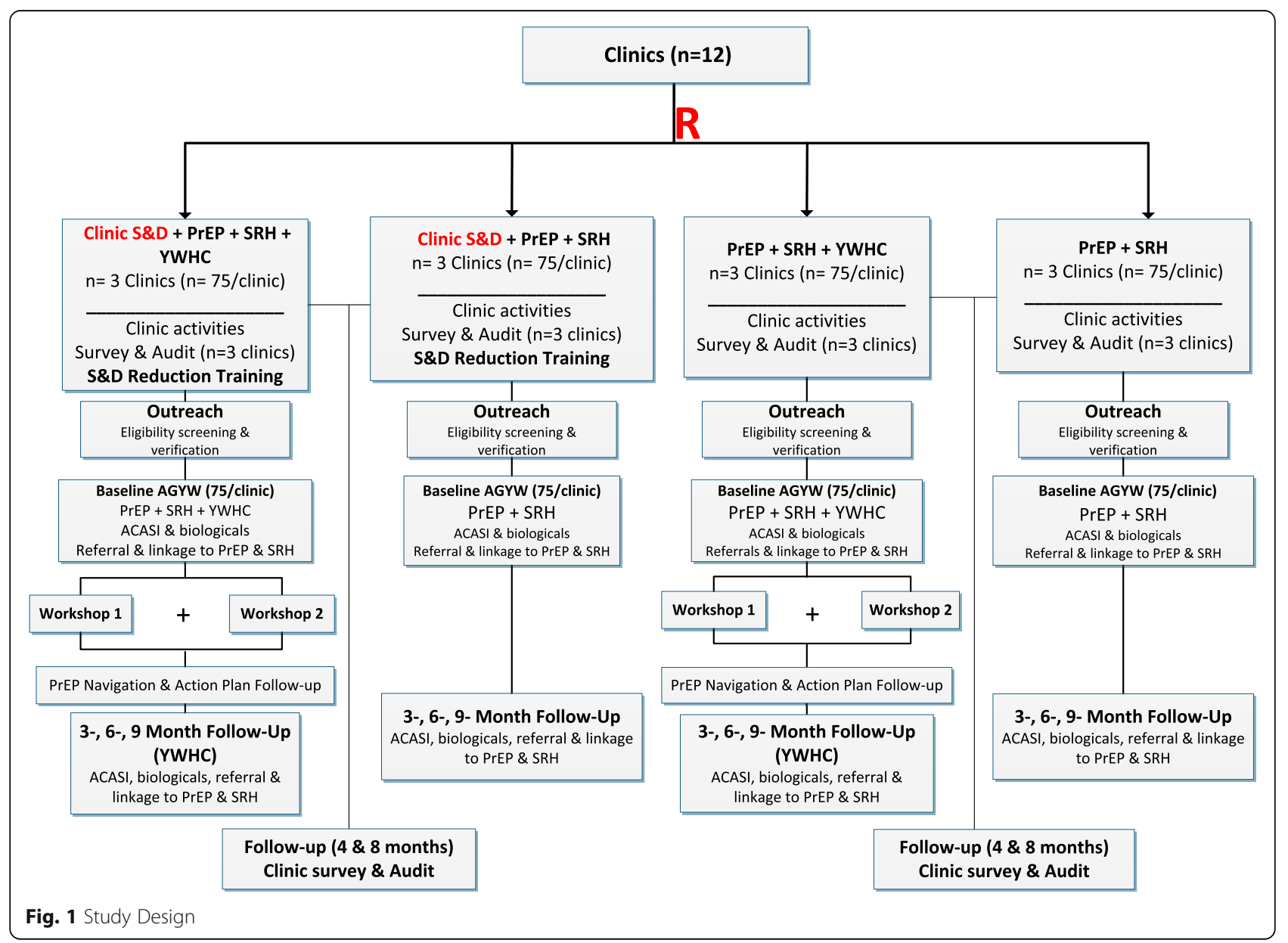


each stratum, clinics were then randomly assigned to study conditions based on a 1:1:1:1 allocation. Because there may be important differences pertinent to the outcomes that cannot be made homogenous, such as geographical region, stratified randomization was conducted to achieve even distribution of these factors across study arms. This reduces variability in the estimation of the intervention effect, with resulting increases in power/ precision of estimates [49].

The six clinics randomized to receive the S\&D reduction training will participate in workshops aimed to reduce clinic staff's S\&D attitudes and behaviors toward AGYW. For the 6 clinics randomized to the YWHC, AGYW participants recruited from communities in clinic catchment areas will be asked to participate in two workshops.

\section{Study procedures}

\section{Data collection: clinic level}

At baseline, all clinics (those receiving training and those receiving no training) complete a clinician (e.g., physicians, nurses) or clinic support staff (e.g., receptionists, clerks) survey assessing (after providing informed consent) SRH knowledge and service provision, attitudes toward PrEP and AGYW seeking PrEP, and observations of stigmatizing and discriminatory behavior in their clinic toward AGYW seeking services for SRH, HIV, and PrEP (see Clinician Questionnaire \& Support Staff Questionnaire in Supplementary Files $1 \& 2$ ). This survey is re-administered at 4- and 8-months post baseline. Given the staff turnover and availability and to ensure to anonymity, staff surveys are not linked across time points; consequently, changes at the clinic level are assessed. Clinic support staff surveys have been translated into a local language, Setswana, for easier comprehension. A clinical audit is also being conducted at baseline and 4month and 8-month follow-up by research study staff to collect information on service utilization, including the number of AGYW receiving health services (including HIV, birth control, and antenatal care) from clinical records; PrEP prescriptions from pharmacy and study nurse records; availability of birth control methods; presence of clinic guidelines and standards of practice for serving sexually active AGYW; working hours that are convenient for AGYW, as well as adequate staff to provide youth friendly services. This audit is conducted across all study clinics.

\section{Stigma and discrimination reduction training}

After baseline data collection, marketing and planning, including the introduction of the training, are proposed for clinics randomized to receive the $S \& D$ reduction training so that time is allocated and protected for staff to attend the workshops. Although all clinic staff members are eligible and encouraged to attend, this study relies on clinic managers to approve who, among those interested, can take part in the assessments and training, as some clinic operations need to be maintained during this time. The HPP S\&D reduction curriculum encourages all staff at all levels to be involved in the training workshops [41] because they are likely to interact with AGYW. For example, in South Africa, support staff such as receptionists and clerks often interact with AGYW because they serve as gatekeepers to clinicians.

The S\&D reduction training incorporates important baseline findings for relevance and ownership through participatory activities addressing key drivers of clinic stigma toward AGYW seeking PrEP and birth control. This includes building awareness of AGYW stigma-its causes, forms and manifestations-reflecting on one's own stigmatizing experiences and attitudes towards AGYW and developing a plan/pledge with actionable steps to reduce S\&D in the clinic. The HPP curriculum $[41,42]$ has been adapted with respect to time, target audience, and type of stigma relevant to this study. Modifications include examples of AGYW seeking services in pictorial displays and other pictures to elicit conversation in the workshops to address various types of bias (see Table 1 for an overview of the S\&D reduction training).

\section{Implementation of stigma and discrimination training}

The S\&D reduction training workshops are conducted by clinic staff who have been selected and trained by experienced $S \& D$ reduction trainers to facilitate these workshops. Preferably, the appointed facilitators are clinic staff who work with youth, such as youth-friendly nurses, and are interested in implementing the S\&D

Table 1 Overview of the S\&D Reduction Training

\begin{tabular}{cl}
\hline Workshop 1 & \\
1 & Opening activities (warmup games, songs, energizers) \\
2 & Naming stigma through pictures \\
3 & Our experience as the stigmatizer and the stigmatized \\
4 & Confidentiality and stigma \\
Workshop 2 & \\
1 & Breaking the sex ice \\
2 & Fears about prescribing pre-exposure prophylaxis (PrEP) \\
Workshop 3 & \\
1 & Panel discussion with adolescent girls and young women \\
2 & Challenge the stigma-and be the change! \\
3 & The blame game: Things people say \\
4 & Stigma-free services for adolescents and young people \\
5 & Writing a code of practice and action plan
\end{tabular}


reduction training workshops. They are supported by YAB members and research study staff and observed by the S\&D reduction master trainers. The clinic S\&D reduction training has 6 modules, with added clinic wide booster training workshops to account for clinic staff turnover.

\section{Data collection: adolescent girls and young women level Recruitment}

AGYW are recruited in communities that are in the study clinic's catchment area. Recruitment is conducted through research study staff, who may also be assisted by trained WBOTs associated with the study clinics. However, screening is only conducted and performed by research study staff. Recruitment is conducted via street outreach at identified areas where AGYW are known to frequent. Street outreach is an established recruitment method that has been used successfully in previous research studies [50-53], including Tshwane WHC studies to reach and recruit a high number of women most at risk of HIV. Study staff visit these hotspots regularly to establish a known presence in the community and gain rapport with community members. Recruitment also is conducted through referrals from the clinic or other staff who conduct HIV and/or pregnancy testing in the community, such as HIV and AIDS, STI, and TB (HAST) counselors.

Participant eligibility criteria include the following: (1) identify as female; (2) HIV negative status; (3) between ages 16 and 24 years; (4) have had condomless sex in the past 3 months with a male partner; (5) not currently pregnant and do not want to get pregnant within the next year; (6) interested in taking a daily pill to prevent HIV (PrEP); (7) not having previously participated in the formative phase of the study; (8) not previously or not currently participating in any other PrEP-related project or research study; (9) not previously or not currently participating in any other HIV study in Tshwane; (10) not on multidrug-resistant tuberculosis (MDR-TB) treatment; (11) lives in one of the target communities; (12) intends to stay in the Tshwane district for the next 12 months; (13) agrees to provide contact information; (14) willing to undergo rapid HIV testing; and (15) willing to undergo pregnancy testing.

Because of the eligibility requirement of an HIVnegative and nonpregnant status, interested potential participants must first consent to rapid testing for HIV and pregnancy to confirm eligibility. Additionally, coenrollment in another HIV study is also checked and any individual who is currently enrolled is excluded.

\section{Screening}

Verbal consent is obtained to screen the potential participant and determine eligibility. Initial eligibility screening of potential participants is performed individually and in private by research study staff. After the initial screening, the next step is to confirm that the participant is HIV-negative, not pregnant, and not enrolled in another HIV study; this is done at the study clinic. Potential participants provide informed consent before conducting these tests. All participants receive a rapid HIV test and a pregnancy test. The only exception is if they have tested negative for HIV the same day and they have an acceptable proof of their HIV testing result, such as a clinic card. If a potential participant cannot be seen for the baseline appointment on the same day as initial screening, they are not tested for HIV and pregnancy until the day of their scheduled baseline appointment to minimize repeat testing. They are rescreened using the initial eligibility screening tool to ensure they meet study eligibility criteria on the day of enrollment.

\section{Consent or assent}

The intake process takes place within the compound of the study clinic, typically in an outdoor tent because of the limited physical space in clinics. Written informed consent or assent is obtained from eligible participants before study enrollment. For potential participants who are younger than 18, consent is required from their mother or a trusted adult woman at least 25 years old who may serve in loco parentis ("in place of a parent"). Parental waiver has been requested to protect a participant's confidentiality if they are uncomfortable having their mother consent for them. This approach has been used successfully in our previous South African studies with adolescents $[19,51]$. In loco parentis enables the participant to select a female adult (either identified by the participant themselves or by the study staff) to provide consent on her behalf.

Once the adult woman who will provide consent is identified, study staff schedule an appointment for the potential participant and the adult to sign the mother/in loco parentis consent. The potential participant is screened separately again and assented separately from the adult woman to prevent coercion, maintain confidentiality, and provide the opportunity for the participant to decline. The adult woman is also required to sign a confidentiality agreement form as part of the consent process so they understand that their experience at the project site is confidential and that they must maintain confidentiality regarding the person for whom they consented.

\section{Intake assessment}

After consenting/assenting, participants also sign a release of medical records permitting the release of information on their SRH, PrEP screening and monitoring 
results, and other referrals and care pertinent to the study.

Participants take a breathalyzer test to detect recent alcohol use (conducted earlier in the appointment because of the limited detection window for alcohol breath scans) and provide urine for drug screening, locator information is collected, and a photograph of the participant is taken to identify the participant for subsequent appointments. This photo is returned or shredded at the final appointment. The participant then completes a baseline questionnaire on a computer tablet via audio computer-assisted self-interview (ACASI) in either English or Setswana (see AGYW Health CoOp Questionnaire in Supplementary File 3). Trained field staff are available to assist the participant at any time and provide referrals based on prompts related to self-reported violence, symptoms of psychological distress, or suicidal ideation experienced in the past 3 months that are triggered as the participant completes the questionnaire. The questionnaire is derived from components of the youth-specific modification of the Revised Risk Behavior Assessment (RRBA) [54], which has been adapted and modified for many studies in South Africa. The modified RRBA for this study contains sections on PrEP knowledge, contraception and other SRH services access and utilization, sexual communication, STI symptoms, alcohol and other drug use, relationship equity and sexual control, economic dependence, personal agency, psychological distress, victimization, and peers and social support. After completing the questionnaire, urine drug screening results are provided to assess the recent use of amphetamine, methamphetamine, benzodiazepine, cocaine, marijuana, opioids, and MDMA. These screening tests are repeated at 3-, 6-, and 9-month follow-up assessments.

After the intake appointment, participants interested in initiating PrEP are referred to the youth-friendly nurse in their respective study clinic or the study's roaming nurse to screen for PrEP eligibility using the National Department of Health (NDoH) guidelines. Baseline screening for PrEP involves reporting of no MDR-TB (part of the study eligibility criteria), HIV and pregnancy testing (conducted prior to the intake process to confirm HIV-negative and not pregnant), estimation of creatinine clearance, and hepatitis B virus (HBV) screening. Contraindications for PrEP use includes poor renal function (estimated creatinine clearance $<60 \mathrm{~mL} / \mathrm{min}$ ) and absence of the hepatitis B surface antigen and antibody. Presence of HBV is not a contraindication for PrEP; however, liver function monitoring is advised if PrEP is initiated. Participants not eligible for PrEP are linked to the public health clinic to receive the standard of care for each respective medical condition. Individuals who are not eligible for PrEP can still remain enrolled in the study.
Participants may decide to initiate PrEP at any time during the study. However, those who do not initiate PrEP during their baseline appointment have to repeat all PrEP eligibility screening. Participants who decide to initiate PrEP are counselled on the importance of adherence and using other protective strategies for up to 20 days of daily dosing before protective levels in vaginal tissue are achieved. Participants are also given guidance on proper management of mild side effects that may occur after initiation and are advised to continue to use condoms and birth control to protect themselves against other STIs and unplanned pregnancy, as PrEP does not provide this type of protection.

Clinic visits to test for HIV and pregnancy status are scheduled 1 month after initiation of PrEP and every 3 months thereafter. At 6 months post initiation of PrEP, screening of creatinine levels is conducted to estimate creatine clearance. If a participant tests positive for HIV at any point in the follow-up appointments, PrEP is stopped for that participant and an active referral is provided for test and treat according the South African HIV guidelines. Nonclinical behavioral study data collection follow-up visits continue to occur at 3, 6, and 9 months post enrollment regardless of whether or when PrEP is initiated or when a participant's PrEP clinic visits take place.

\section{Sexual and reproductive health services}

All participants who complete their baseline appointment are actively referred to the designated youthfriendly nurse or youth-friendly service at their respective study clinic for SRH services; specifically, birth control services. Follow-up on these linkages is conducted at the 3-month follow-up appointment for participants in the control groups and during monthly check-ins for participants in the YWHC groups.

\section{YWHC workshops}

The YWHC workshops comprise a 2-session, 4-module program designed to build on the nexus of substance use, HIV, and GBV by increasing knowledge, skills, and agency to reduce GBV and substance use, and ways to decrease sexual risk and HIV incidence. Previous research in South Africa has shown positive outcomes with the WHC, including that women in the WHC were more likely to use condoms with their boyfriends during their last episode of sexual intercourse, more likely to use female condoms with their boyfriends in the past month, more likely to negotiate condom use, and less likely to report daily substance use at follow-up than women who were not in the $\mathrm{WHC}[38,39,52]$. The workshops also include voices and quotes from the formative focus group discussion participants (see Table 2 for an overview of the workshops). Typically, workshops 
are conducted approximately 7 to 10 days apart, but can be completed on the same day and in either order because of limited availability of the participants; for example, some participants are full-time students. Both workshops end with participants developing an individualized and personalized risk-reduction action plan with assistance from staff. Also, any relevant referrals are offered. Participants also receive toiletry kits and pill boxes with a colorful bag to discreetly store their PrEP. Once both workshops are completed, participants complete a Satisfaction Form to provide feedback and suggestions regarding the intervention. Staff conduct further followup with participants individually to check in on their personal action plans at least monthly via mobile phone or in person.

\section{PrEP navigation after PrEP initiation}

Although the South African National Strategic Plan for HIV, TB and STIs (2017-2022) [47] outlines the availability of PrEP for all AGYW who are HIV-negative and at risk of HIV, PrEP roll-out in public health clinics has yet to occur. Consequently, a majority of public health clinic staff have not been trained on PrEP prescribing and dispensing protocols. Prior to the national roll-out, the PrEPARE Project partnered with the $\mathrm{NDoH}$ to train

Table 2 Overview of the YWHC Workshops

Workshop 1
Topics
- Becoming an adult woman and influences
- Sex, sexual expectations, and risk
- Our reproductive bodies (female and male anatomy)
- STIs and HIV
- Ways to reduce risk
- Male and female condoms
- Ways of communicating, negotiating, and problem-solving
- PrEP
- Birth control
- Action plan (complete Workshop 1 goals and steps in workshop booklet)
Workshop 2
Topics
- Inequality and gender power
- Becoming strong women and concern for how boyfriends treat you
- Abuse and violence
- Safety tips for going out
- Alcohol and drug use
- Parenting
- The importance of education and goals
- Social support, especially taking PrEP
- Action plan (complete Workshop 2 goals and steps in workshop booklet)

youth-friendly nurses and pharmacists charged with supporting and implementing PrEP delivery from all study clinics on standardized PrEP protocols. A roaming nurse has been hired to assist in the management and support of dispensing PrEP where there is a shortage of trained clinic staff.

PrEP navigation, which occurs through phone calls and text messaging, provides participants in the YWHC intervention arms who are on PrEP with much-needed support, specifically in the first 1 to 2 weeks of PrEP initiation where many participants may experience minor side effects that often lead to PrEP discontinuation. Study staff work with participants to develop feasible plans that can support the daily use of PrEP, such as the pill boxes they receive, adherence strategies, social support, and addressing other concerns about PrEP. Participants also are reminded about their upcoming refill and check-up appointments. This navigation occurs throughout the study duration as participants are due for their refills.

\section{Follow-up assessments}

Participants in both study arms return for their 3-, 6and 9-month behavioral and biological follow-up assessments. Research staff track participants using locator information provided during the intake appointment to help retain participants. The follow-up visit includes reconsenting; updating locator information; a follow-up questionnaire via ACASI; and biological testing for HIV, pregnancy, alcohol use (breathalyzer), and other recent drug use. Dried blood spots (DBS) also are collected at these appointments from participants who report to be on PrEP. The PrEP medication used for this study is a combination of FTC $200 \mathrm{mg} / \mathrm{TDF} 300 \mathrm{mg}$ tablets. The DBS are used to assess the presence of tenofovir diphosphate (TFV-DP), a measure of cumulative and recent adherence, using a previously validated methodology [55].

\section{Data management and quality assurance}

To protect confidentiality, the study assigns each participant a unique alphanumeric study participant identification number (PID). This PID is the only link between the behavioral and biological data and the identifying information collected for locating participants for their follow-up interviews. Locator forms, consent/assent agreements, and any data that can be linked through the PID are stored separately from other data in doublelocked file cabinets in locked rooms at the study's project site, with restricted access.

Data collection for this study is conducted by highly trained staff from the community who develop a rapport with the study participants to engender trust and elicit the most accurate data possible. All staff sign a confidentiality agreement and are trained on the study's Quality 
Assurance Protocol and Quality Management Plan (QMP). Study data are encrypted before they are transmitted daily from the field site to secure servers in the United States. The US-based data manager reviews additional automated quality control checks that the software generates each day. If any critical inconsistencies are noted, the data manager contacts the project director and the field supervisor to resolve these inconsistencies. The Principal Investigator, other members of the research team, and the field staff receive daily field activity reports.

\section{Data and safety monitoring plan and data and safety monitoring board}

Procedures have been put in place to address adverse events (AEs) or serious adverse events (SAEs), such as improper disclosure of information or mental or emotional discomfort. As specified in our Data and Safety Monitoring Plan (DSMP), SAEs are reported to the Principal Investigator and the South African CoInvestigator/Medical Director within $24 \mathrm{~h}$ of an occurrence and to the Data and Safety Monitoring Board (DSMB), the funding agency, and the Institutional Review Board within $72 \mathrm{~h}$, with appropriate action taken immediately. The study does not interfere with any activities or reports that are part of the public health clinics' standard operating procedures that do not directly affect the study participants adversely. The DSMP ensures that procedures have been set in place to safeguard the security, validity, and integrity of study data, and that study staff are trained on the policies and procedures for data management according to the Quality Assurance Protocol and QMP. The DSMP also outlines the data analysis plan including preliminary analyses of data for quality assurance and to track the progress of the study.

The study established a DSMB comprising three members: a psychologist, an infectious disease clinician, and a bioethicist. The DSMB will meet every 6 months during the trial to review study progress and ensure adherence to the DSMP. This board is independent from the researchers and the study sponsoring institution. Board members discussed whether stopping rules were necessary for this study; they determined that stopping rules were not necessary.

\section{Outcomes}

The clinic-level primary outcomes for this study include clinic staff attitudes and environment, observed discrimination, and stigmatizing avoidance behaviors toward AGYW, as measured by staff surveys and the clinic audits. The AGYW-level primary outcomes include the level of PrEP readiness, uptake, and adherence (as measured by self-report and DBS), and SRH uptake. The
AGYW-level secondary outcomes include frequency of substance use, as measured by the RRBA and biological drug screening and breathalyzer tests to assess recent alcohol use; GBV, assessed through self-reported experiences of emotional, physical and sexual abuse; sexual risk as measured by condomless sex, impaired sex, other sex partners; self-reported frequency of experienced stigma.

\section{Sample size and power}

For the AGYW analyses, the total sample size and number of clusters (clinic catchment areas) were selected to ensure sufficient power to detect meaningful differences in our primary outcomes, while also balancing considerations tied to reducing possible contamination (exposure to the intervention in control clusters) and implementation feasibility with a set number of clinics in the study area. Sample size estimates for tests of two proportions in a cluster-randomized design [56] were conducted for a 2 -sided test with significance level of 0.05 , power of .80 , and intra-cluster correlation of 0.01 (based on our team's past research in Cape Town communities), assuming $10 \%$ attrition over the 9-month follow-up period $[52,57]$. This was not adjusted for multiple comparisons. Based on these parameters and calculations, the study sample includes 12 clusters (defined as clinics and their catchment areas), with a total sample size of 900 AGYW enrolled, 75 per cluster (clinic catchment area). This sample was chosen to ensure that meaningful differences in the primary outcomes of PrEP readiness, uptake, and adherence between groups would be detectable. We are powered to detect a difference between 9 to $13 \%$ in primary outcomes. Power analyses were first conducted in PASS software [58] and were refined in Stata [59] (see PrEPARE Additional Power Calculation Information in Supplementary File 4).

\section{Analysis}

Analysis of primary outcome measures will determine whether the provision of $S \& D$ reduction training for clinic staff and the YWHC intervention for AGYW will increase PrEP readiness, PrEP uptake, and PrEP adherence, and SRH uptake.

\section{Clinic level}

AGYW service utilization will be assessed and analyzed through clinic level data at each of the 12 study clinics during an 8-month period pre- and post-training (e.g., HIV testing; number of PrEP prescriptions). Baseline and follow-up clinic staff surveys will be analyzed to examine changes in attitudes and stigma at the clinic level. Initial analyses will be descriptive and evaluate group differences comparing intervention and control clinics using unpaired $t$-tests with unequal variance or 
nonparametric tests, as appropriate. Using multilevel modeling approach, we will investigate and account for clustering at the clinic level. We will use difference-indifferences (DD) methods as done in another South African study to examine utilization of services by AGYW and staff attitudes and behaviors in the 8 months between intervention and control clinics [60]. The DD estimator reflects the average change in clinics that received $S \& D$ reduction training, after the average change in utilization in control clinics is subtracted, assuming parallel trends in clinics over time had the S\&D reduction training not been implemented. DD estimates will be calculated for each outcome separately; models will include covariates for time (pre- vs. post-intervention), treatment (training vs. no training) and an interaction between the two. The clinics identified are similar in size, services, and populations served. Any clinic features for which we cannot achieve balance through the design will be included as covariates in the multivariable DD analyses. A stigma score will constitute one primary outcome for these analyses. To examine short-term changes between pre-intervention and 4-month follow-up, DD methods can be used to compare the average change in stigma scores in clinics that received S\&D reduction training, removing any changes observed in control clinics. We will use generalized estimating equations (GEE) [61] to examine provider-level stigma scores measured at 4- and 8-month follow-up comparing trained and untrained clinics, accounting for clustering at the clinic-level.

\section{AGYW level}

The testing of Aim 3 will be done by intention to treat analysis, examining the effects of intervention groups at both levels (clinic and individual) on PrEP readiness, uptake and adherence. PrEP uptake will be defined as any use of PrEP. We will consider both short-term uptake within the first 3 months after study enrollment and uptake at any point during the 9-month follow-up period. Initially, we will calculate the proportion of AGYW with any use of PrEP, overall, and within each study arm, calculating 95\% confidence intervals using the binomial distribution. Given the need to consider the influence of the cluster randomization by clinic, we will use generalized estimating equations (GEE) [61] models with a logit link and an exchangeable correlation structure to estimate the effects of randomization groups on both short-term (3-month follow-up) and long-term follow-up period (9month follow-up). Models will include both intervention groups (i.e., S\&D reduction training and YWHC) as covariates. Effects of clustering by randomization clinic may be examined by adjusting the variance by the inflation factor $[1+(m-1) r]$, where $m$ is the average clinic size and $r$ is the interclass correlation estimate. If the Hausman assumption of correlation between the random and fixed effects is violated, then we may include fixed effects representing cluster identification.

Finally, we will include additional baseline covariates in the models, including sociodemographic factors and behavioral risks, should initial descriptive analyses suggest differences in the distribution of these factors across study arms. In subsequent exploratory analyses, we will examine the potential mediating and moderating roles of key behavioral, social, and structural factors hypothesized to influence PrEP uptake (e.g., social support; economic dependence), as outlined in Outcomes. To assess adherence in the past month, we will measure drug-level concentration, TFV-DF assessed through DBS samples, at 3, 6 and 9 months. The threshold for adherence will be established based on recommendations from ongoing studies and research designed to inform thresholds in women. Secondary adherence measures will include selfreported use, which will be examined as the number of days in the past 30 days when PrEP was taken. Using clinical/pharmacy data on PrEP dispensation and selfreport of months in which PrEP was used, we will examine adherence (the number of months of PrEP use) and discontinuation. Initially, we will examine differences in the proportions of AGYW who are adherent at months 3,6 , and 9 in each study arm, calculating confidence intervals using a binomial distribution. Our analytic approaches will be similar to those described for uptake. We will use generalized linear mixed models to allow for multiple variance components, including clustering of clinics and repeated measures of adherence within individuals over time. We will use logit, Poisson, and linear regression approaches, as appropriate.

Analyses of secondary outcomes will use similar analytic approaches and examine hypotheses tied to intervention effects on behavioral risk endpoints (e.g., substance use) and on AGYW's assessment of stigma (e.g., participants attending the S\&D reduction trained clinics will report lower levels of stigma from clinic staff than participants who attend the non-trained clinics). Other model specifications, such as zero-inflated Poisson or negative binomial will be considered for count outcomes (e.g., the number of condomless sex acts) that have excess zero values or overdispersion.

From the AGYW outcomes, it is hypothesized that AGYW enrolled from clinics who received clinic-level S\&D reduction training will have higher PrEP and SRH utilization than AGYW enrolled from clinics that did not receive training. AGYW enrolled from clinics that are randomized to receive the YWHC intervention will have higher PrEP readiness, uptake and adherence than those enrolled from clinics that are randomized to PrEP and SRH provision only. AGYW enrolled from communities in clinic catchment areas that are randomized to 
receive the YWHC will report less HIV-related risk at follow-up (e.g., GBV, substance use, condomless sex) than those enrolled from clinics that are randomized to PrEP and SRH provision only.

Missing data in terms of data management may occur because of nonresponse and study attrition. We will analyze differential attrition in relation to participants' key demographic characteristics. Based on our prior research conducted in South Africa with AGYW who engage in sex risk behaviors, we estimate an average retention rate of $90 \%$ over the repeated follow-ups [19, $37,62]$. We will address missingness by including demographic covariates that will serve as proxies for dropout and by conducting a sensitivity analysis.

\section{Ethical approval}

\section{Full experimental protocol approval and amendments}

The experimental phase of the study was approved by the South African Medical Association Research Ethics Committee (SAMAREC), which serves as the Institutional Review Board (IRB) of Record, in addition to the Tshwane District Health Research Committee and the Skills Development for Tshwane Municipal Clinics. Since receiving approval to conduct the full study, we amended the protocol to improve the study design and procedures. Table 3 summarizes these approvals.

With COVID-19, there have been barriers and disruptions to PrEP initiation and dispensing, which may alter some of the expected study outcomes, study activities and data collection. We attempted to address these unprecedented circumstances (as noted in Table 3) without compromising the rigor of the research design and ensuring that participants were able to stay on PrEP. Some of the strategies included getting personal protective equipment, ensuring a travel nurse was able to dispense PrEP, and when possible, providing refills longer than 30 days. We will be monitoring these circumstances as they are changing daily.

\section{Dissemination}

Study findings will be disseminated to participants, health care professionals, and other stakeholders through our established $\mathrm{YAB}$ and $\mathrm{CCB}$, program newsletter, journal articles, conference presentations and also other targeted dissemination channels.

\section{Discussion}

This article describes the study protocol for a research study aimed at evaluating the impact of a stigma and discrimination reduction training program for clinic staff and the efficacy of a multilevel HIV prevention strategy that addresses young women's empowerment to include substance use, sexual risk, and GBV; and access to and use of SRH and PrEP.

The UNAIDS Fast-Track Approach agenda, which drives the 95-95-95 goals for HIV prevention and treatment by 2030, includes ambitious targets for reducing new HIV infections and achieving zero discrimination, including in healthcare settings [1]. To meet this agenda, we must reduce the incidence of HIV among AGYW who are the least likely to have access to HIV prevention, testing, and treatment services but carry the greatest burden of HIV. Furthermore, although PrEP is safe and is a highly effective prevention method, especially among individuals at high risk of HIV acquisition, PrEP uptake and adherence remains low among certain high

Table 3 Summary of Protocol Approval and Amendments

\begin{tabular}{|c|c|}
\hline $\begin{array}{l}\text { Date of } \\
\text { approval }\end{array}$ & Protocol and Amendments \\
\hline $1 / 2019$ & Initial submission to conduct the experimental phase of the study \\
\hline $3 / 2019$ & Request for SAMAREC to be the IRB of Record \\
\hline $3 / 2019$ & Revised research protocol and supporting materials to prepare for the full study phase \\
\hline 4/2019 & Amended full study research activities to allow for the provision of PrEP \\
\hline $7 / 2019$ & Addition of participant follow-up consent documents and the 3-month follow-up questionnaire \\
\hline $10 / 2019$ & $\begin{array}{l}\text { Minor revisions to study screener and eligibility criteria to ensure participants are not currently enrolled in another HIV project and } \\
\text { to the study protocol regarding follow-up on pregnancy outcomes of participants who become pregnant during the course of the } \\
\text { study }\end{array}$ \\
\hline $11 / 2019$ & Reduced the number of clinic staff follow-up visits from 3 to 2 appointments—-specifically, 4- and 8-month follow-up appointments \\
\hline $2 / 2020$ & $\begin{array}{l}\text { Minor modifications to study documents and protocol to prevent co-enrollment of participants involved in other HIV studies in the } \\
\text { Tshwane area }\end{array}$ \\
\hline $3 / 2020$ & $\begin{array}{l}\text { Modification to allow project staff to complete at-home data entry of de-identified data, and the submission of a staff agreement of } \\
\text { confidentiality for at-home data entry because of the COVID-19 lockdown }\end{array}$ \\
\hline $4 / 2020$ & Minor modifications to clinician and support staff follow-up questionnaires and consenting procedures \\
\hline $4 / 2020$ & Notification to the IRB of resumption of limited face-to-face appointment with study participants during the COVID-19 lockdown \\
\hline $4 / 2020$ & Submission of the COVID-19 questionnaire to be administered via telephone during the COVID-19 lockdown \\
\hline
\end{tabular}


priority populations, particularly AGYW. This is the first study of its kind in South Africa to attempt to address structural S\&D in public health clinics as a way to improve PrEP readiness, uptake, and adherence among this population and in turn reduce HIV burden.

The study comprises some notable innovations: (1) developing a partnership with the South African Department of Health to reduce S\&D by clinic staff; (2) engaging the study's $Y A B$ and the $C C B$ in the adaptation of the YWHC (YAB) and the S\&D (CCB) interventions, study marketing materials (YAB), $S \& D$ reduction training (YAB) as stakeholders to share their voices, and the regular meetings. This is an important step towards involving community members in the dissemination and implementation of HIV-prevention structural interventions. Support for structural changes may increase when the people whom the intervention is likely to affect participate in the process [63]; and (3) other contextual factors, such as GBV and substance use, and using an evidence-based, gender-focused approach are addressed. Additionally, partnering with the $\mathrm{NDoH}$ and the public health clinics is facilitating capacity building through the standardized PrEP provider training that will be required when PrEP provision is scaled up to a population level. Because this study is being conducted in collaboration with public health clinics, it may bring to light challenges that need to be addressed prior to integrating PrEP delivery into public health facilities, such as workload, the need for task shifting or sharing, and the need to enhance PrEP's general acceptance in usual care settings.

Few interventions currently exist that address the multiple levels of the psychosocial and community barriers that prevent AGYW from accessing healthcare services. The adapted YWHC provides a more comprehensive HIV prevention toolkit with PrEP and reproductive health as next generation science. If found to be efficacious, the YWHC plus addressing the structural S\&D may help reduce barriers to HIV prevention among a key population with the greatest incidence-offering more hope for an HIV-free generation of young women.

\section{Trial status}

Recruiting.

\section{Supplementary information}

Supplementary information accompanies this paper at https://doi.org/10. 1186/s12889-020-09458-y.

Additional file 1: Figure File 1. CONSORT Diagram.

Additional file 2: Supplementary File 1. Clinician baseline survey.

Additional file 3: Supplementary File 2. Support staff baseline survey. Additional file 4: Supplementary File 3. AGYW baseline survey.
Additional file 5: Supplementary File 4. Estimated Detectable Differences in PrEP Uptake between AGYW in the PrEP/SRH + YWHC arm vs. PrEP/SRH only, controlling for intervention arm of clinic.

Additional file 6: Supplementary File 5. Spirit checklist.

Additional file 7: Supplementary File 6. Ethical approval proofs.

\section{Abbreviations}

ACASI: Audio computer-assisted self-interviewing; AE: Adverse event; AGYW: Adolescent girls and young women; ARV: Antiretroviral; CCB: Community Collaborative Board; DBS: Dried blood spots; DD: Difference-in-differences; DREAMS: Determined, Resilient, Empowered, AIDS-free, Mentored and Safe ; DSMB: Data and Safety Monitoring Board; DSMP: Data and Safety Monitoring Plan; FTC: Emtricitabine; GBV: Genderbased violence; GEE: Generalized estimating equations; HAST: HIV/AIDS, STI, and TB; HBV: Hepatitis B virus; HPLC: High-performance liquid

chromatography; HPP: Health Policy Project; IRB: Institutional Review Board; MDMA: 3,4-Methylenedioxymethamphetamine; MDR-TB: Multi-drug resistant tuberculosis; NDoH: National Department of Health; PID: Participant identification number; PrEP: Pre-exposure prophylaxis; PrEPARE: PRevention, Empowering, and PRotEcting; QMP: Quality Management Plan; RRBA: Revised Risk Behavior Assessment; SAE: Serious adverse event; SAMAREC: South African Medical Association Research Ethics Committee; S\&D: Stigma and discrimination; SRH: Sexual and reproductive health; STI: Sexually transmitted infection; TB: Tuberculosis; TDF: Tenofovir disoproxil fumarate; TFV-

DP: Tenofovir diphosphate; UNAIDS: Joint United Nations Programme on HIV/AIDS; WBOT: Ward-based outreach team; WHC: Women's Health CoOp; YAB: Youth Advisory Board; YWHC: Young Women's Health CoOp

\section{Acknowledgements}

We would like to acknowledge the editorial support from Jeffrey Novey.

\section{Authors' contributions}

All authors participated in the article preparation and protocol planning. WMW conceived of the study, oversaw drafts, and finalized the manuscript; $F A B$, JN contributed to drafting the manuscript; AMM, LN, ISS, BNH contributed to writing and reviewing the manuscript; $C P B, B M$ and $K A$ reviewed the manuscript and provided feedback for finalization. All authors read and approved the final manuscript.

\section{Funding}

Research reported in this publication was supported by the Eunice Kennedy Shriver National Institute of Child Health \& Human Development of the National Institutes of Health under Award Number R01HD094629 (PI: Wechsberg). The content is solely the responsibility of the authors and does not necessarily represent the official views of the National Institutes of Health. The Eunice Kennedy Shriver National Institute of Child Health \& Human Development of the National Institutes of Health had no role in study design, data collection and analysis, decision to publish, or preparation of the manuscript.

\section{Availability of data and materials}

The study is currently enrolling; therefore, data collection is ongoing. The datasets used and/or analyzed during the current study will be available from the corresponding principal investigator when outcomes and publications are complete.

\section{Ethics approval and consent to participate}

This study has been approved by the South African Medical Association Research Ethics Committee (SAMAREC), which serves as the Institutional Review Board (IRB) of Record. In addition, the Tshwane Research Committee and the Skills Development for Tshwane Municipal Clinics reviewed and approved this study. Written consent will be obtained from all participants.

Consent for publication

Not applicable.

Competing interests

The authors declare that they have no competing interests. 


\section{Author details}

'Substance Use, Gender, and Applied Research Program, RTI International, 3040 E. Cornwallis Road, Research Triangle Park, NC 27709-2194, USA. ${ }^{2}$ Maternal and Child Health, University of North Carolina Gillings School of Global Public Health, 135 Dauer Drive, Chapel Hill, NC 27599, USA. ${ }^{3}$ Department of Psychology, North Carolina State University, 640 Poe Hall, Campus Box 7650, Raleigh, NC 27695, USA. ${ }^{4}$ Psychiatry and Behavioral Sciences, Duke University School of Medicine, 40 Duke Medicine Circle, Durham, NC 27710, USA. ${ }^{5}$ Health Behavior, University of North Carolina Gillings School of Global Public Health, 135 Dauer Drive, Chapel Hill, NC 27599, USA. ${ }^{6}$ Center for Global Health, RTI International, 2150 Shattuck Avenue, Suite 800, Berkeley, CA 94704, USA. 'Epidemiology Division, Berkeley School of Public Health, University of California, 2121 Berkeley Way, Room 5302, Berkeley, CA 94720, USA. ${ }^{8}$ International Global Health Division, International Development Group, RTI International, 701 13th Street NW \#750, Washington, DC 20005, USA. ${ }^{9}$ Alcohol and Drug Abuse Research Unit, Medical Research Council Francie van Zijl Drive, Parow Valley, Cape Town, South Africa. ${ }^{10}$ Setshaba Research Centre, 2088 Block H, Soshanguve 0152, South Africa.

Received: 6 July 2020 Accepted: 27 August 2020

Published online: 15 September 2020

\section{References}

1. UNAIDS (Joint United Nations Programme on HIV/AIDS). UNAIDS data 2019. Geneva: UNAIDS; 2019. Available from: https:/www.unaids.org/sites/default/ files/media_asset/2019-UNAIDS-data_en.pdf.

2. Simbayi LC, Zuma K, Zungu N, Moyo S, Marinda E, Jooste S, et al. South African national HIV prevalence, incidence, behaviour and communication survey, 2017. Cape Town: HSRC Press; 2019. Available from: http://www.hsrc. ac.za/uploads/pageContent/10779/SABSSM\%20V.pdf.

3. Cunningham SD, Kerrigan DL, Jennings JM, Ellen JM. Relationships between perceived STD-related stigma, STD-related shame and STD screening among a household sample of adolescents. Perspect Sex Reprod Health. 2009:41(4):225-30.

4. Myers B, Carney T, Wechsberg WM. "Not on the agenda": a qualitative study of influences on health services use among poor young women who use drugs in Cape Town, South Africa. Int J Drug Policy. 2016;30:52-8.

5. Nyblade L, Stockton M, Nyato D, Wamoyi J. Perceived, anticipated and experienced stigma: exploring manifestations and implications for young people's sexual and reproductive health and access to care in NorthWestern Tanzania. Cult Health Sex. 2017;19(10):1092-107.

6. Wechsberg WM, Ahmed K, Ndirangu J, Browne FA, Bonner C, Speizer I, et al. Can We End HIV Among Adolescent Girls and Young Women in South Africa? Key Challenges with PrEP. Philadelphia: American Public Health Association Annual Meeting; 2019.

7. Wechsberg W, Ndirangu J, Browne F, Peasant Bonner C, Nyblade L, Minnis $A$, et al. Addressing stigma and gender inequalities as antecedents to ending AIDS with adolescent women: who has the power? London: AIDS Impact; 2019.

8. Karim QA, Meyer-Weitz A, Mboyi L, Carrara H, Mahlase G, Frohlich JA, et al. The influence of AIDS stigma and discrimination and social cohesion on HIV testing and willingness to disclose HIV in rural KwaZulu-Natal, South Africa. Global Public Health. 2008;3(4):351-65.

9. Musheke M, Ntalasha H, Gari S, McKenzie O, Bond V, Martin-Hilber A, et al. A systematic review of qualitative findings on factors enabling and deterring uptake of HIV testing in sub-Saharan Africa. BMC Public Health. 2013;13(1): 220.

10. Sweeney SM, Vanable PA. The association of HIV-related stigma to HIV medication adherence: a systematic review and synthesis of the literature. AIDS Behav. 2016;20(1):29-50.

11. Turan JM, Bukusi EA, Onono M, Holzemer WL, Miller S, Cohen CR. HIV/AIDS stigma and refusal of HIV testing among pregnant women in rural Kenya: results from the MAMAS study. AIDS Behav. 2011;15(6):1111-20.

12. Calabrese SK, Dovidio JF, Tekeste M, Taggart T, Galvao RW, Safon CB, et al. HIV pre-exposure prophylaxis stigma as a multidimensional barrier to uptake among women who attend Planned Parenthood. J Acquir Immune Defic Syndr. 2018;79(1):46-53.

13. Amico KR, Wallace M, Bekker LG, Roux S, Atujuna M, Sebastian E, et al. Experiences with HPTN 067/ADAPT study-provided open-label PrEP among women in Cape Town: facilitators and barriers within a mutuality framework. AIDS Behav. 2017:21(5):1361-75.

14. Pintye J, Beima-Sofie KM, Makabong OP, Njoroge A, Trinidad SB, Heffron RA, et al. HIV-uninfected Kenyan adolescent and young women share perspectives on using pre-exposure prophylaxis during pregnancy. AIDS Patient Care STDs. 2018:32(12):538-44.

15. Vazquez L, Moll AP, Kacin A, Ndlovu NE, Shenoi SV. Perceptions of HIV Preexposure prophylaxis among young pregnant women from rural KwaZulu-Natal, South Africa. AIDS Patient Care STDS. 2019;33(5):214-9.

16. Velloza J, Khoza N, Scorgie F, Chitukuta M, Mutero P, Mutiti K, et al. The influence of HIV-related stigma on PrEP disclosure and adherence among adolescent girls and young women in HPTN 082: a qualitative study. I Int AIDS Soc. 2020;23(3):e25463.

17. Turan JM, Elafros MA, Logie CH, Banik S, Turan B, Crockett KB, et al. Challenges and opportunities in examining and addressing intersectional stigma and health. BMC Med. 2019;17(1):7.

18. Rao D, Elshafei A, Nguyen M, Hatzenbuehler ML, Frey S, Go VF. A systematic review of multi-level stigma interventions: state of the science and future directions. BMC Med. 2019;17(1):41.

19. Carney T, Browne FA, Myers B, Kline TL, Howard B, Wechsberg WM. Adolescent female school dropouts who use drugs and engage in risky sex: effects of a brief pilot intervention in Cape Town, South Africa. AIDS care. 2019;31(1):77-84.

20. Saul J, Bachman G, Allen S, Toiv NF, Cooney C, Beamon T. The DREAMS core package of interventions: a comprehensive approach to preventing HIV among adolescent girls and young women. PLoS One. 2018;13(12):e0208167.

21. Hanscom B, Janes HE, Guarino PD, Huang Y, Brown ER, Chen YQ, et al. Brief report: preventing HIV-1 infection in women using Oral Preexposure prophylaxis: a meta-analysis of current evidence. J Acquir Immune Defic Syndr. 2016;73(5):606-8.

22. Thomson KA, Baeten JM, Mugo NR, Bekker LG, Celum CL, Heffron R. Tenofovir-based oral preexposure prophylaxis prevents HIV infection among women. Curr Opin HIV AIDS. 2016;11(1):18-26.

23. CDC Statement on FDA Approval of Drug for HIV Prevention [press release]. Atlanta, Georgia: Centers for Disease Control and Prevention (CDC), July 2012. Available from: https://www.cdc.gov/nchhstp/newsroom/2012/fdaapprovesdrugstatement.html.

24. Medicines Control Council approves fixed-dose combination of tenofovir disoproxil fumarate and emtricitabine for pre-exposure prophylaxis of HIV [press release]. 3 December 2015. Available from: http://www.mccza.com/ documents/2e4b3a5310.11_Media_release_ARV_FDC_PrEP_Nov15_v1.pdf.

25. Corneli A, Namey E, Ahmed K, Agot K, Skhosana J, Odhiambo J, et al. Motivations for reducing other HIV risk-reduction practices if taking preexposure prophylaxis: findings from a qualitative study among women in Kenya and South Africa. AIDS Patient Care STDs. 2015;29(9):503-9.

26. Fladseth K, Gafos M, Newell ML, McGrath N. The impact of gender norms on condom use among HIV-positive adults in KwaZulu-Natal, South Africa. PLoS One. 2015;10(4):e0122671.

27. Teitelman AM, Jemmott JB, Bellamy SL, Icard LD, O'Leary A, Heeren GA, et al. Partner violence, power, and gender differences in south African adolescents' HIV/sexually transmitted infections risk behaviors. Health Psychol. 2016;35(7):751-60.

28. Pettifor A, Nguyen NL, Celum C, Cowan FM, Go V, Hightow-Weidman L. Tailored combination prevention packages and PrEP for young key populations. J Int AIDS Soc. 2015;18(2 Suppl 1):19434.

29. Bekker LG, Gill K, Wallace M. Pre-exposure prophylaxis for south African adolescents: what evidence? S Afr Med J. 2015;105(11):907-11.

30. Corneli AL, Deese J, Wang M, Taylor D, Ahmed K, Agot K, et al. FEM-PrEP: adherence patterns and factors associated with adherence to a daily oral study product for pre-exposure prophylaxis. J Acquir Immune Defic Syndr. 2014;66(3):324-31.

31. van der Straten A, Brown ER, Marrazzo JM, Chirenje MZ, Liu K, Gomez K, et al. Divergent adherence estimates with pharmacokinetic and behavioural measures in the MTN-003 (VOICE) study. J Int AIDS Soc. 2016;19(1):20642.

32. Marrazzo JM, Ramjee G, Richardson BA, Gomez K, Mgodi N, Nair G, et al. Tenofovir-based preexposure prophylaxis for HIV infection among African women. N Engl J Med. 2015;372(6):509-18.

33. Celum CL, Delany-Moretlwe S, Baeten JM, van der Straten A, Hosek S, Bukusi EA, et al. HIV pre-exposure prophylaxis for adolescent girls and young women in Africa: from efficacy trials to delivery. J Int AIDS Soc. 2019; 22(Suppl 4):e25298. 
34. Hodges-Mameletzis I, Fonner VA, Dalal S, Mugo N, Msimanga-Radebe B, Baggaley R. Pre-exposure prophylaxis for HIV prevention in women: current status and future directions. Drugs. 2019;79(12):1263-76.

35. Wechsberg WM, Lam WK, Zule WA, Bobashev G. Efficacy of a womanfocused intervention to reduce HIV risk and increase self-sufficiency among African American crack abusers. Am J Public Health. 2004;94(7):1165-73.

36. Wechsberg WM, Bonner CP, Zule WA, van der Horst C, Ndirangu J, Browne FA, et al. Addressing the nexus of risk: biobehavioral outcomes from a cluster randomized trial of the Women's health CoOp plus in Pretoria, South Africa. Drug Alcohol Depend. 2019;195:16-26.

37. Wechsberg WM, Jewkes R, Novak SP, Kline T, Myers B, Browne FA, et al. A brief intervention for drug use, sexual risk behaviours and violence prevention with vulnerable women in South Africa: a randomised trial of the Women's Health CoOp. BMJ Open. 2013;3(5):e002622.

38. Wechsberg WM, Zule WA, Luseno WK, Kline TL, Browne FA, Novak SP, et al. Effectiveness of an adapted evidence-based woman-focused intervention for sex workers and non-sex workers: the Women's health CoOp in South Africa. J Drug Issues. 2011;41(2):233-52.

39. Wechsberg WM, Luseno WK, Lam WK, Parry CD, Morojele NK. Substance use, sexual risk, and violence: HIV prevention intervention with sex workers in Pretoria. AIDS Behav. 2006;10(2):131.

40. Wechsberg WM, Browne FA, Zule WA, Novak SP, Doherty IA, Kline TL, et al. Efficacy of the young Women's CoOp: an HIV risk-reduction intervention for substance-using African-American female adolescents in the south. J Child Adolesc Subst Abuse. 2017;26(3):205-18

41. Kidd R, Clay S, Stockton M, Nyblade L. Facilitator's training guide for a stigma-free health facility. Washington, DC: Futures Group, Health Policy Project; 2015.

42. Nyblade L, Addo NA, Atuahene K, Alsoufi N, Gyamera E, Jacinthe S, et al. Results from a difference-in-differences evaluation of health facility HIV and key population stigma-reduction interventions in Ghana. J Int AIDS Soc. 2020;23(4):e25483.

43. Nyblade L, Mbuya-Brown R, Sabasaba AN, Ezekiel M, Kiwia P, Stewart C, et al. Understanding and responding to stigma and discrimination in health facilities in Tanzania. Policy brief. Washington, DC: Health Policy Plus; 2018. Available from: http://www.healthpolicyplus.com/ns/pubs/10267-10481 TanzaniaStigmat.pdf.

44. Nyblade L, Stewart C, Kiwia P, Manyama W, Mbuya-Brown R, Bowsky S. Towards stigma-free health facilities in Tanzania: guide for trainers. Washington, DC: Health Policy Plus; 2019.

45. Ekstrand ML, Raj T, Heylen E, Nyblade L, Devdass D, Pereira M, et al. Reducing HIV stigma among healthcare providers in India using a partly tablet-administered intervention: the DriSti trial. AIDS Care. 2020;32(sup2): 14-22.

46. Nyblade L, Srinivasan K, Mazur A, Raj T, Patil DS, Devadass D, et al. HIV stigma reduction for health facility staff: development of a blendedlearning intervention. Front Public Health. 2018:6:165.

47. South African National AIDS Council (SANAC). Let our actions count: South Africa's National Strategic Plan for HIV, TB and STIs (2017-2022). Pretoria: SANAC; 2017. Available from: https://sanac.org.za/wp-content/uploads/2017/ 06/NSP_FullDocument_FINAL.pdf.

48. Crespi CM. Improved designs for cluster randomized trials. Annu Rev Public Health. 2016:37(1):1-16.

49. Mdege ND, Brabyn S, Hewitt C, Richardson R, Torgerson DJ. The $2 \times 2$ cluster randomized controlled factorial trial design is mainly used for efficiency and to explore intervention interactions: a systematic review. J Clin Epidemiol. 2014;67(10):1083-92.

50. Wechsberg WM, Ndirangu JW, Speizer IS, Zule WA, Gumula W, Peasant C, et al. An implementation science protocol of the Women's health CoOp in healthcare settings in Cape Town, South Africa: a stepped-wedge design. BMC Womens Health. 2017;17(1):85

51. Wechsberg WM, Browne FA, Carney T, Myers B, Minnis A, MacDonald R, et al. The young Women's health CoOp in Cape Town, South Africa: study protocol for a cluster-randomised trial for adolescent women at risk for HIV. BMC Public Health. 2018;18(1):859.

52. Wechsberg WM, van der Horst C, Ndirangu J, Doherty IA, Kline T, Browne FA, et al. Seek, test, treat: substance-using women in the HIV treatment cascade in South Africa. Addict Sci Clin Pract. 2017:12(1):12

53. Wechsberg WM, Zule WA, Ndirangu J, Kline TL, Rodman NF, Doherty IA, et al. The biobehavioral Women's health CoOp in Pretoria, South Africa: study protocol for a cluster-randomized design. BMC Public Health. 2014;14: 1074

54. Wechsberg WM. Revised risk behavior assessment, part I and part II. Research Triangle Park: Research Triangle Institute; 1998.

55. Anderson PL, Liu AY, Castillo-Mancilla JR, Gardner EM, Seifert SM, McHugh C, et al. Intracellular Tenofovir-Diphosphate and Emtricitabine-Triphosphate in Dried Blood Spots following Directly Observed Therapy. Antimicrob Agents Chemother. 2018;62(1):e01710-7.

56. Donner A, Klar N. Design and analysis of cluster randomization trials in Health Research. London: Arnold; 2000.

57. Wechsberg WM, Zule WA, El-Bassel N, Doherty IA, Minnis AM, Novak SD, et al. The male factor: outcomes from a cluster randomized field experiment with a couples-based HIV prevention intervention in a south African township. Drug Alcohol Depend. 2016;161:307-15.

58. PASS. 14.0 .8 ed: NCSS LLC; 2017.

59. STATA. 16 ed. College Station: StataCorp LLC; 2019

60. McGovern ME, Herbst K, Tanser F, Mutevedzi T, Canning D, Gareta D, et al. Do gifts increase consent to home-based HIV testing? A difference-indifferences study in rural KwaZulu-Natal, South Africa. Int J Epidemiol. 2016; 45(6):2100-9.

61. Hanley JA, Negassa A, Edwardes MD, Forrester JE. Statistical analysis of correlated data using generalized estimating equations: an orientation. Am J Epidemiol. 2003;157(4):364-75.

62. Myers B, Carney T, Browne FA, Wechsberg WM. A trauma-informed substance use and sexual risk reduction intervention for young south African women: a mixed-methods feasibility study. BMJ Open. 2019;9(2): e024776.

63. Reed SJ, Miller RL. The adolescent medicine trials network for HIVAI. The benefits of youth engagement in HIV-preventive structural change interventions. Youth Soc. 2014;46(4):529-47.

\section{Publisher's Note}

Springer Nature remains neutral with regard to jurisdictional claims in published maps and institutional affiliations.

Ready to submit your research? Choose BMC and benefit from

- fast, convenient online submission

- thorough peer review by experienced researchers in your field

- rapid publication on acceptance

- support for research data, including large and complex data types

- gold Open Access which fosters wider collaboration and increased citations

- maximum visibility for your research: over $100 \mathrm{M}$ website views per year

At BMC, research is always in progress.

Learn more biomedcentral.com/submissions 